\title{
Current and universal scaling in anomalous transport
}

\author{
I. Goychuk, ${ }^{1}$ E. Heinsalu, ${ }^{1,2,3}$ M. Patriarca, ${ }^{1}$ G. Schmid, ${ }^{1}$ and P. Hänggi ${ }^{1}$ \\ ${ }^{1}$ Institut für Physik, Universität Augsburg, Universitätsstr. 1, D-86135 Augsburg, Germany \\ ${ }^{2}$ Institute of Theoretical Physics, Tartu University, 4 Tähe Street, 51010 Tartu, Estonia \\ ${ }^{3}$ Fachbereich Chemie, Philipps-Universität Marburg, 35032 Marburg, Germany
}

(Dated: August 31, 2006)

\begin{abstract}
Anomalous transport in tilted periodic potentials is investigated within the framework of the fractional Fokker-Planck dynamics and the underlying continuous time random walk. The analytical solution for the stationary, anomalous current is obtained in closed form. We derive a universal scaling law for anomalous diffusion occurring in tilted periodic potentials. This scaling relation is corroborated with precise numerical studies covering wide parameter regimes and different shapes for the periodic potential, being either symmetric or ratchet-like ones.
\end{abstract}

PACS numbers: 02.50.Ey, 05.40.Fb, 05.60.Cd

Over recent years we witness an increasing interest in dynamical processes that occur in systems exhibiting anomalous diffusive behavior, possessing prominent interdisciplinary applications that range from physics and chemistry to biology and medicine 1]. The benchmark of anomalous diffusion is the occurrence of a mean square displacement of the form $\left\langle\delta r^{2}(t)\right\rangle \sim t^{\alpha}$, where $\alpha \neq 1$. Depending on the anomalous diffusion exponent $\alpha$ the motion can either be subdiffusive $(0<\alpha<1)$ or superdiffusive $(\alpha>1)$.

In the following we focus on the subdiffusive regime. Examples for subdiffusive transport are very diverse, encompassing phenomena such as charge carrier transport in amorphous semiconductors, nuclear magnetic resonance, diffusion in percolative and porous systems, transport on fractal geometries and dynamics of a bead in a polymeric network, as well as protein conformational dynamics 1, 2, 3]. Another topic that recently gained attention is the transport of Brownian particles in the presence of a periodic force, relevant in Josephson junctions, rotating dipoles in external fields, superionic conductors, charge density waves, synchronization phenomena, diffusion on crystal surfaces, particle separation by electrophoresis, and biophysical processes such as intracellular transport [4, 5, 6].

With this work, we present intriguing results for anomalous diffusion and transport under the combined action of a periodically varying spatial force and an external constant bias $F$. In particular, we derive a closed form expression in terms of two quadratures for the fluctuation-assisted current and its corresponding nonlinear mobility. Furthermore, we establish a universal scaling relation for diffusive transport which is valid in tilted, corrugated nonlinear periodic potentials.

We start out by presenting a novel derivation of the fractional Fokker-Planck equation (FFPE) from a space-continuous limit of a continuous-time random walk (CTRW). Our derivation involves nearest neighbors jumps only; moreover, it provides new insight and complements prior treatments in Refs. 1, 7, 8].

Derivation of the FFPE from the CTRW. We study a CTRW characterized by the probability distributions $\psi_{i}(\tau)$ for the residence times $\tau$, considering only jumps between nearest-neighbor sites on a one dimensional lattice $\left\{x_{i}\right\}$, with lattice period $\Delta x$. Such a CTRW is described by a generalized master equation (GME) for the site populations $P_{i}(t)$, reading $[9,10,11]$

$$
\begin{aligned}
\dot{P}_{i}(t) & =\int_{0}^{t}\left\{K_{i-1}^{+}\left(t-t^{\prime}\right) P_{i-1}\left(t^{\prime}\right)+K_{i+1}^{-}\left(t-t^{\prime}\right) P_{i+1}\left(t^{\prime}\right)\right. \\
& \left.-\left[K_{i}^{+}\left(t-t^{\prime}\right)+K_{i}^{-}\left(t-t^{\prime}\right)\right] P_{i}\left(t^{\prime}\right)\right\} \mathrm{d} t^{\prime},
\end{aligned}
$$

where the Laplace-transform of the kernel $K_{i}^{ \pm}(t)$ is related to the Laplace-transform of the residence time distribution (RTD) via $\tilde{K}_{i}^{ \pm}(s)=q_{i}^{ \pm} s \tilde{\psi}_{i}(s) /\left[1-\tilde{\psi}_{i}(s)\right]$. The quantities $q_{i}^{ \pm}$are the splitting probabilities to jump from site $i$ to site $i \pm 1$, obeying $q_{i}^{+}+q_{i}^{-}=1$.

Choosing for the RTD the Mittag-Leffler distribution,

$$
\psi_{i}(\tau)=-\frac{\mathrm{d}}{\mathrm{d} \tau} E_{\alpha}\left[-\left(\nu_{i} \tau\right)^{\alpha}\right]
$$

one obtains $\tilde{K}_{i}^{ \pm}(s)=q_{i}^{ \pm} \nu_{i}^{\alpha} s^{1-\alpha}$. Here $E_{\alpha}(z)=$ $\sum_{n=0}^{\infty} z^{n} / \Gamma(n \alpha+1)$ denotes the Mittag-Leffler function and the quantity $\nu_{i}^{-1}$ is the time-scaling parameter at site $i$. The corresponding GME can be recast as a fractional master equation (FME) [12, 13], reading

$$
\begin{aligned}
\dot{P}_{i}(t) & ={ }_{0} \hat{D}_{t}^{1-\alpha}\left\{f_{i-1} P_{i-1}(t)+g_{i+1} P_{i+1}(t)\right. \\
& \left.-\left(f_{i}+g_{i}\right) P_{i}(t)\right\}
\end{aligned}
$$

where the symbol ${ }_{0} \hat{D}_{t}^{1-\alpha}$ stands for the integrodifferential operator of the Riemann-Liouville fractional derivative acting on a generic function of time $\chi(t)$, as [1, 2, 14

$$
{ }_{0} \hat{D}_{t}^{1-\alpha} \chi(t)=\frac{1}{\Gamma(\alpha)} \frac{\partial}{\partial t} \int_{0}^{t} \mathrm{~d} t^{\prime} \frac{\chi\left(t^{\prime}\right)}{\left(t-t^{\prime}\right)^{1-\alpha}} .
$$

The quantities $f_{i}=q_{i}^{+} \nu_{i}^{\alpha}$ and $g_{i}=q_{i}^{-} \nu_{i}^{\alpha}$ will be referred to as fractional forward and backward rates. Using the normalization condition for the splitting probabilities one 
obtains that $\nu_{i}=\left(f_{i}+g_{i}\right)^{1 / \alpha}$, and $q_{i}^{+}=f_{i} /\left(f_{i}+g_{i}\right)$ and $q_{i}^{-}=g_{i} /\left(f_{i}+g_{i}\right)$, in terms of the fractional rates. For an arbitrary potential $U(x)$ one can set

$$
\begin{aligned}
& f_{i}=\left(\kappa_{\alpha} / \Delta x^{2}\right) \exp \left[-\beta\left(U_{i+1 / 2}-U_{i}\right)\right], \\
& g_{i}=\left(\kappa_{\alpha} / \Delta x^{2}\right) \exp \left[-\beta\left(U_{i-1 / 2}-U_{i}\right)\right] .
\end{aligned}
$$

Here $U_{i} \equiv U(i \Delta x)$ and $U_{i \pm 1 / 2} \equiv U(i \Delta x \pm \Delta x / 2)$, with $U(x)$ the total potential, $\beta=1 / k_{B} T$ is the inverse of temperature, and $\kappa_{\alpha}$ is the anomalous diffusion coefficient with dimension $\mathrm{cm}^{2} \mathrm{~s}^{-\alpha}$. The form (4) of the fractional rates ensures that the Boltzmann relation is satisfied, $f_{i-1} / g_{i}=\exp \left[\beta\left(U_{i-1}-U_{i}\right)\right]$.

By use of the Laplace-transform method one can show that the FME (3) can be brought into the form 15]

$$
D_{*}^{\alpha} P_{i}(t)=f_{i-1} P_{i-1}(t)+g_{i+1} P_{i+1}(t)-\left(f_{i}+g_{i}\right) P_{i}(t),
$$

where the symbol $D_{*}^{\alpha}$ on the l.h.s. denotes the Caputo fractional derivative [14],

$$
D_{*}^{\alpha} \chi(t)=\frac{1}{\Gamma(1-\alpha)} \int_{0}^{t} \mathrm{~d} t^{\prime} \frac{1}{\left(t-t^{\prime}\right)^{\alpha}} \frac{\partial}{\partial t^{\prime}} \chi\left(t^{\prime}\right) .
$$

Let us introduce the finite difference operator $\Delta / \Delta x$, $\Delta P(x, t) / \Delta x=[P(x+\Delta x / 2, t)-P(x-\Delta x / 2, t)] / \Delta x$, which in the limit $\Delta x \rightarrow 0$ yields the partial derivative operator $\partial / \partial x$. Using the fractional rates (4) the FME (5) can now be rewritten as

$$
D_{*}^{\alpha} P\left(x_{i}, t\right)=\kappa_{\alpha} \frac{\Delta}{\Delta x}\left(e^{-\beta U\left(x_{i}\right)} \frac{\Delta}{\Delta x} e^{\beta U\left(x_{i}\right)} P\left(x_{i}, t\right)\right),
$$

where $P\left(x_{i}, t\right)=P(i \Delta x, t)=P_{i}(t) / \Delta x$. By taking the continuous limit in Eq. (6) one obtains the FFPE,

$$
D_{*}^{\alpha} P(x, t)=\kappa_{\alpha} \frac{\partial}{\partial x}\left(e^{-\beta U(x)} \frac{\partial}{\partial x} e^{\beta U(x)} P(x, t)\right),
$$

which can be rewritten in the well-known form with the Riemann-Liouville fractional derivative on the r.h.s [1, 7],

$$
\frac{\partial P(x, t)}{\partial t}=\kappa_{\alpha}{ }_{0} \hat{D}_{t}^{1-\alpha} \frac{\partial}{\partial x}\left(e^{-\beta U(x)} \frac{\partial}{\partial x} e^{\beta U(x)} P(x, t)\right)
$$

Biased diffusion. For a constant force $F$ the potential reads $U(x)=-F x$ and the fractional rates (4) become site-independent, $f_{i} \equiv f$ and $g_{i} \equiv g$, satisfying the Boltzmann relation, i.e., $f / g=\exp (\beta F \Delta x)$ for any finite value of $\Delta x$. Using the Laplace-transform one finds the solutions of Eq. (5) for the mean particle position and the mean square displacement of anomalous biased Brownian motion [16],

$$
\begin{aligned}
\langle x(t)\rangle & =\langle x(0)\rangle+\Delta x(f-g) t^{\alpha} / \Gamma(\alpha+1), \\
\left\langle\delta x^{2}(t)\right\rangle & =\left\langle\delta x^{2}(0)\right\rangle+\Delta x^{2}(f+g) t^{\alpha} / \Gamma(\alpha+1) \\
+ & \left(\frac{2}{\Gamma(2 \alpha+1)}-\frac{1}{\Gamma^{2}(\alpha+1)}\right) \Delta x^{2}(f-g)^{2} t^{2 \alpha} .
\end{aligned}
$$

With respect to the case of normal diffusion the expression for the mean square displacement contains besides a thermal contribution $\propto t^{\alpha}$ also a ballistic-like term $\propto t^{2 \alpha}$. As a consequence, a value $\alpha<1$ does not necessarily imply subdiffusive behavior. In fact, in the presence of bias for $1 / 2<\alpha<1$ superdiffusion takes place.

For a finite bias $F$, the ballistic term in Eq. (9b) equals zero only in the case $\alpha=1$, for which normal Brownian motion is recovered. From Eqs. (9) one obtains then a generalized nonlinear Einstein relation, which is nonlinear in force and valid for a finite space step $\Delta x$,

$$
\frac{\left\langle\delta x^{2}(t)\right\rangle-\left\langle\delta x^{2}(0)\right\rangle}{\langle x(t)\rangle-\langle x(0)\rangle}=\Delta x \operatorname{coth}(F \beta \Delta x / 2) .
$$

In the limit $F \rightarrow 0$, Eq. (10) yields the well-known Einstein relation $(\alpha=1), \kappa_{\alpha} / \mu_{\alpha}(0)=1 / \beta$, between the thermal diffusion coefficient

$$
\kappa_{\alpha}=\Gamma(\alpha+1) \lim _{t \rightarrow \infty} \frac{\left\langle\delta x^{2}(t)\right\rangle_{F=0}}{2 t^{\alpha}}
$$

and the linear mobility $\mu_{\alpha}(F=0)$, with the nonlinear mobility $\mu_{\alpha}(F)=v_{\alpha}(F) / F$ being related to the anomalous current $v_{\alpha}$ (see below), i.e.

$$
\mu_{\alpha}(F)=\Gamma(\alpha+1) \lim _{t \rightarrow \infty} \frac{\langle x(t)\rangle}{F t^{\alpha}} .
$$

The same Einstein relation is valid also between the submobility and the subdiffusion coefficient for any $\alpha<1$ [17], as the ballistic term in the mean square displacement (9b) vanishes for $F=0$.

However, a relation analogous to the generalized nonlinear Einstein relation (10) ceases to be valid for $\alpha<1$ for any finite $F$, as the mean square displacement becomes dominated by the ballistic contribution in the long time limit. Instead, from Eqs. (9) one obtains the following asymptotic scaling relation,

$$
\lim _{t \rightarrow \infty} \frac{\left\langle\delta x^{2}(t)\right\rangle}{\langle x(t)\rangle^{2}}=\frac{2 \Gamma^{2}(\alpha+1)}{\Gamma(2 \alpha+1)}-1 .
$$

This result no longer contains the fractional transition rates and holds true independent of the strength of the bias $F$ and the temperature $T$. The relation (13) has been obtained in [18] for a continuous-time random walker that is exposed to a constant force. As a main finding of this work we prove below that this very relation holds true universally for the nontrivial case of tilted nonlinearly corrugated potentials.

Tilted periodic potentials: fractional Fokker-Planck current. We next study the case of a periodic potential with period $L$ in the presence of a constant force $F$. Towards this goal, it is convenient to consider the FFPE (7), in which the Caputo derivative appears only on the 1.h.s. Analogously to the case of normal Brownian motion [4, 5, 19] one obtains that the probability flux

$$
J_{\alpha}(x, t)=-\kappa_{\alpha} e^{-\beta U(x)} \frac{\partial}{\partial x} e^{\beta U(x)} P(x, t)
$$


reaches asymptotically the stationary current value, i.e.,

$$
D_{\alpha}^{*}\langle x(t)\rangle=L J_{\alpha}=v_{\alpha}(F)=\mu_{\alpha}(F) F .
$$

The anomalous current $v_{\alpha}(F)$ is given in closed form by

$$
v_{\alpha}(F)=\frac{\kappa_{\alpha} L[1-\exp (-\beta F L)]}{\int_{0}^{L} \mathrm{~d} x \int_{x}^{x+L} \mathrm{~d} y \exp (-\beta[U(x)-U(y)])} .
$$

This result constitutes a first main result: It is the anomalous counterpart of the current known for normal diffusion. It obeys a form that mimics the celebrated Stratonovich formula for normal diffusion with $\alpha=1$ [4, 19, 20]. From Eq. (15) the mean particle position follows as

$$
\langle x(t)\rangle=\langle x(0)\rangle+v_{\alpha}(F) t^{\alpha} / \Gamma(\alpha+1) .
$$

Universal scaling in washboard potentials. We next show that the relation in (13) is valid as well for anomalous transport in washboard-like potentials. We prove this by mapping the dynamics onto an equivalent CTRW; i.e., we consider a discrete state reduction of the continuous diffusion process $x(t)$ : To this aim, we introduce a lattice with sites $\left\{\hat{x}_{j}=j L\right\}$, located at the minima of the periodic part of the potential, and study the RTD $\hat{\psi}_{j}(\tau)$ for the hopping process between sites $\left\{\hat{x}_{j}\right\}$. For such a system, the ratio $\hat{q}_{j}^{+} / \hat{q}_{j}^{-}=\exp (\beta F L)$ equals that of the constant force case, due to the choice $\Delta x=L$. Furthermore, the analogy between the solutions (9a) and (17), both exhibiting an asymptotic power law $\propto t^{\alpha}$, implies the same form $\hat{\psi}_{j}(\tau) \propto 1 / \tau^{1+\alpha}$ for $\tau \rightarrow \infty$. In fact, for $\hat{\psi}_{j}(\tau) \sim \alpha \hat{\nu}_{j}^{-\alpha} / \Gamma(1-\alpha) \tau^{1+\alpha}$, with some suitable scaling coefficients $\hat{\nu}_{j}$, the corresponding kernels of the GME obey $\tilde{K}_{j}^{ \pm}(s)=\hat{q}_{j}^{ \pm} \hat{\nu}_{j}^{\alpha} s^{1-\alpha}$ in the limit $s \rightarrow 0$. Therefore, by making use of Tauberian theorems for the Laplacetransform [11], it follows that the asymptotic solution $(t \rightarrow \infty)$ is of the form (9), being determined only by the asymptotic power law behavior of the RTD [21], despite the fact that the values of $\hat{f}$ and $\hat{g}$ depend on the chosen shape for the periodic potential. Because the result in (13) is independent of $\hat{f}$ and $\hat{g}$, the scaling relation thus still holds true. It is universal in the sense that it holds independently of the detailed shape of the washboard potential, the temperature $T$ and the bias strength $F$.

Numerical verification of universal scaling. We have numerically tested the scaling relation (13) and the generalized Stratonovich formula (16) through the simulation of the fractional CTRW in tilted washboard potentials of various shapes and for different parameter values for $\alpha, F$, and dimensionless $T$. In doing so, we not only investigate the archetype case of a symmetric simple cosine potential $U_{1}(x)=\cos (x)$, but as well a symmetric double hump potential $U_{2}(x)=\cos (x)+\cos (2 x)$ and an asymmetric, ratchet-like potential $U_{3}(x)=\sin (x)+\sin (2 x) / 3$.

In Fig. 1 we depict the scaled nonlinear mobility $k_{B} T \mu_{\alpha}(F) / \kappa_{\alpha}$ defined by Eq. (12) for the cosine potential. The force is in units of the critical tilt $F_{\text {cr }}$, which corresponds to the disappearance of potential minima and

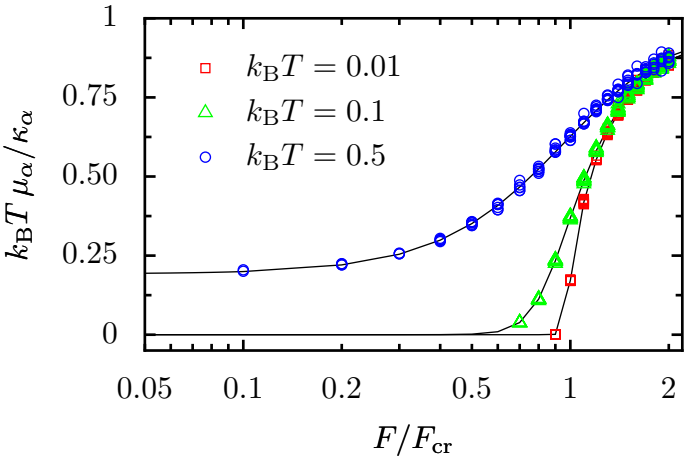

FIG. 1: (color online). The scaled nonlinear mobility $k_{B} T \mu_{\alpha}(F) / \kappa_{\alpha}$ is depicted for the case of a tilted cosine potential versus $F / F_{\text {cr }}$. The numerics for different temperatures $T$ and different $\alpha$-values, varying between $0.1-1$, fits the analytic prediction (16) (continuous lines) within the statistical errors.

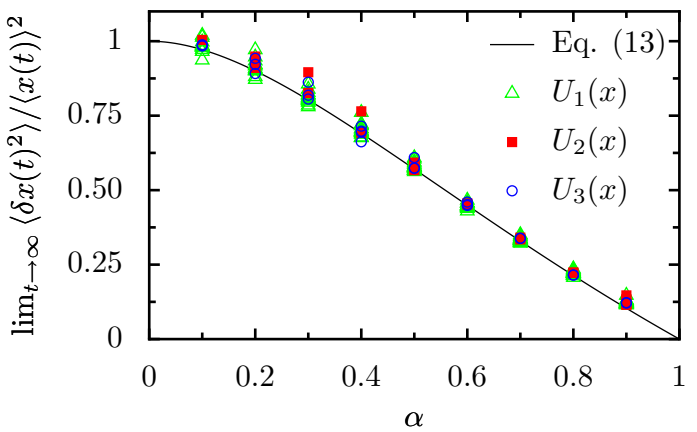

FIG. 2: (color online). Universal scaling: Asymptotic values of the ratio $\left\langle\delta x^{2}(t)\right\rangle /\langle x(t)\rangle^{2}$ as a function of the parameter $\alpha$ for anomalous diffusion. All the points corresponding to the same $\alpha$ but different values of $F$ and $T$ match the function given in Eq. (13) (solid line) within the statistical errors. We use three different temperatures: $T=0.01$ with the bias $F$ ranging between $0.9-2.0$; correspondingly, $T=0.1, F=$ $0.7-2.0$ and $T=0.5, F=0.4-2.0$. The open triangles correspond to the cos-potential $U_{1}(x)$, the filled squares to the double-hump potential $U_{2}(x)$ and the open circles to the asymmetric ratchet potential $U_{3}(x)$, see in text.

maxima. For a given temperature $T$, all values of $\mu_{\alpha}$ with $\alpha$ taken from the interval, $0.1-1$, coincide with Eq. (16) (continuous lines). For tilting forces that exceed $F / F_{c r}=2$ the dynamics approaches the behavior of a free CTRW being exposed to a constant bias. We further note that the regime of linear response at low temperatures is numerically not accessible. This is so because in this parameter regime the corresponding escape times governing the anomalous fluctuation-assisted transport become far too large [20].

The universal scaling in tilted corrugated periodic potentials is illustrated with Fig. 2 in which the asymptotic ratio $\left\langle\delta x^{2}(t)\right\rangle /\langle x(t)\rangle^{2}$ is plotted versus $\alpha$ for the three different periodic potentials mentioned above. For a given $\alpha$ various data are presented, corresponding to different 
potential shapes and values of $F$ and $T$. As one can deduce, these points overlap, demonstrating that the ratio is independent of bias and temperature, as well as the specific shape of $U(x)$. At the same time, the data fit very well with the analytical expression (13) (continuous line).

As detailed above, the long time behavior of the system is determined only by the tail of the RTD. Since we are interested in the asymptotic behavior $(t \rightarrow \infty)$, we have used in the numerical simulations the Pareto distribution $(0<\alpha<1)$,

$$
\psi_{i}(\tau)=\frac{\alpha b \nu_{i}}{\left(1+b \nu_{i} \tau\right)^{1+\alpha}}=-\frac{\mathrm{d}}{\mathrm{d} \tau} \frac{1}{\left(1+b \nu_{i} \tau\right)^{\alpha}} .
$$

This distribution, with $b=\Gamma(1-\alpha)^{1 / \alpha}$, has precisely the same asymptotic form as the Mittag-Leffler distribution (2). For $\alpha=1$ corresponding to a normal Brownian process we have employed the exponential RTD $\psi_{i}(\tau)=$ $\nu_{i} \exp \left(-\nu_{i} \tau\right)$. In our simulations we have assumed a space step $\Delta x \ll L$ such that $U^{\prime \prime}(x) \Delta x \ll 2 U^{\prime}(x)$, ensuring the smoothness of the periodic potential. Each trajectory was assigned the same initial condition $x\left(t_{0}\right)=x_{0}$. Then a residence time $\tau$ was extracted randomly from the RTD (18), time was increased to $t_{1}=t_{0}+\tau$ and the particle was moved either to the right or left site with respective probabilities $q_{i}^{+}$and $q_{i}^{-}$. Using this procedure we computed the full random trajectory of the Brownian particle $\left(10^{3}\right.$ time steps at least). The mean displacement and the mean square displacement were obtained as averages over $10^{4}$ trajectories.

Conclusions. We have investigated the CTRW with power-law distributed residence times in a periodic potential in the presence of an external bias. The fractional Fokker-Planck dynamics has been derived from the corresponding space-inhomogeneous CTRW in a novel manner. The celebrated Stratonovich solution for the stationary current in a tilted periodic potential has been generalized to the case of anomalous transport. Moreover, we have proven that there exists a universal scaling law (13) - relating the mean square displacement and the mean particle position in washboard potentials - that does not involve the exact form of the periodic potential, the applied bias $F$, and the temperature $T$. This universal scaling has been verified by numerical simulations.

Our findings for the current and the mean square fluctuations can readily be applied to the diverse physical situations mentioned in the introduction. The versatile and widespread use of the grand Stratonovich result for the stationary current in case of normal diffusion can thus readily be put to powerful use in all those multifaceted applications where the corresponding transport behaves anomalously.

We thank E. Barkai, J. Klafter, R. Metzler and S. Denysov for constructive discussions. This work has been supported by the ESF STOCHDYN project and the Estonian Science Foundation through grant no. $5662(\mathrm{EH})$, the DFG via research center, SFB-486, project A10, the Volkswagen Foundation, via project no. I/80424.
[1] R. Metzler, J. Klafter, Phys. Rep. 339, 1 (2000).

[2] I.M. Sokolov, J. Klafter, A. Blumen, Phys. Today 55 (11), 48 (2002).

[3] H. Yang et al., Science 302, 262 (2003); S.C. Kou, X.S. Xie, Phys. Rev. Lett. 93, 180603 (2004).

[4] H. Risken, The Fokker-Planck Equation (SpringerVerlag, Berlin, 1996); P. Hänggi, H. Thomas, Phys. Rep. 88, 207 (1982).

[5] P. Reimann, Phys. Rep. 361, 57 (2002); R. D. Astumian, P. Hänggi, Phys. Today 55 (11), 33 (2002).

[6] P. Hänggi, F. Marchesoni, F. Nori, Ann. Phys. (Leipzig) 14, 51 (2005).

[7] R. Metzler, E. Barkai, J. Klafter, Phys. Rev. Lett. 82, 3563 (1999); E. Barkai, Phys. Rev. E 63, 46118 (2001).

[8] R. Metzler, E. Barkai, J. Klafter, Europhys. Lett. 46, 431 (1999); E. Barkai, R. Metzler, J. Klafter, Phys. Rev. E 61, $132(2000)$

[9] V.M. Kenkre, E.W. Montroll, M.F. Shlesinger, J. Stat. Phys. 9, 45 (1973); A.I. Burshtein, A.A. Zharikov, S.I. Temkin, Theor. Math. Phys. 66, 166 (1986); I. Goychuk, Phys. Rev. E 70, 16109 (2004).

[10] B.D. Hughes, Random Walks and Random Environments, Vol. 1: Random Walks (Clarendon Press, Oxford, 1995).

[11] G.H. Weiss, Aspects and Applications of the Random Walk (North-Holland, Amsterdam, 1994).
[12] I.M. Sokolov, R. Metzler, Phys. Rev. E 67, 010101(R) (2003); A.A. Stanislavsky, Phys. Rev. E 67, 21111 (2003).

[13] I. Goychuk, P. Hänggi, Phys. Rev. E 70, 51915 (2004).

[14] R. Gorenflo, F. Mainardi, in: Fractals and Fractional Calculus in Continuum Mechanics, edited by A. Carpinteri, F. Mainardi (Springer, Wien, 1997), pp. 223-276.

[15] R. Hilfer, L. Anton, Phys. Rev. E 51, R848 (1995).

[16] R. Metzler, J. Klafter, I.M. Sokolov, Phys. Rev. E 58, 1621 (1998).

[17] E. Barkai, V.N. Fleurov, Phys. Rev. E 58, 1296 (1998).

[18] M. Shlesinger, J. Stat. Phys. 10, 421 (1974); H. Scher, E.W. Montroll, Phys. Rev. E 12, 2455 (1975).

[19] R.L. Stratonovich, Radiotekhnika i elektronika 3 (No. 4), 497 (1958); V.I. Tikhonov, Avtomatika i telemekhanika 20 (No. 9), 1188 (1959); R.L. Stratonovich, Topics in the Theory of Random Noise, Vol. II (Gordon and Breach, New York-London, 1967); Yu.M. Ivanchenko and L.A. Zil'berman, Zh. Eksp. Teor. Fiz. 552395 (1968) [Sov. Phys. JETP 28, 1272 (1969)]; V. Ambegaokar, B.I. Halperin, Phys. Rev. Lett. 22, 1364 (1969).

[20] P. Hänggi, P. Talkner, M. Borkovec, Rev. Mod. Phys. 62, 251 (1990).

[21] F. Mainardi, A. Vivoli, R. Gorenflo, Fluct. Noise Lett. 5, L291 (2005). 